\title{
Localización óptima y confiable de instalaciones en una cadena de suministro
}

\author{
Optimal and reliable facility location in a supply chain
}

\author{
Fernando González-Solano $^{1 *} \quad$ Juan Escorcia-Caballero ${ }^{1} \quad$ Luis Patiño-Toledo ${ }^{1}$ \\ Recibido 2 de diciembre de 2015, aceptado 21 de noviembre de 2016 \\ Received: December 2, 2015 Accepted: November 21, 2016
}

\begin{abstract}
RESUMEN
En el diseño de las cadenas de suministro es necesario tener en cuenta factores económicos y no económicos. En esta investigación se analizan los costos de transporte, los costos fijos de abrir un centro de consolidación y la fiabilidad de la cadena de suministro, con el fin de determinar la ubicación óptima de las instalaciones que minimizan tanto la probabilidad de fracaso como los costos totales de la cadena. El objetivo es diseñar cadenas de suministro robustas que serán capaces de absorber los impactos negativos causados por eventos disruptivos, como huracanes, tornados, terremotos, las protestas laborales, accidentes, entre otros. Para lograr este objetivo, se diseñó un modelo de programación entera no lineal que determina la ubicación óptima de los medios bajo los criterios de coste y fiabilidad. El modelo propuesto fue validado con datos obtenidos de la agroindustria del departamento del Atlántico, Colombia. Los resultados muestran una mejora del 9,5\% en la fiabilidad con un aumento del 9,8\% del coste total del transporte de la cadena de suministro; sin embargo, en el modelo propuesto las empresas no tendrían que incurrir en gastos de adecuación de los centros de consolidación, teniendo en cuenta que las instalaciones actuales tienen la capacidad para convertirse en centro de consolidación sin incurrir en costos significativos.
\end{abstract}

Palabras clave: Confiabilidad, evento disruptivo, cadenas de suministro, resiliencia, optimización.

\begin{abstract}
In this paper, we study facility location problem under many optimization criteria, as minimize the probability of failure and the supply chain total cost. We incorporate account transportation costs, consolidation center cost and reliability of the supply chain. The approach is to design robust supply chains that will be able to absorb negative impacts caused by disruptive events, such as hurricanes, twisters, earthquakes, strikes, accidents, among others. We provide a nonlinear integer-programming model that determines the optimal location of facilities under the criteria of cost and reliability. The proposed model was validated with data obtained from a supply chain of agribusiness sector of Atlantic department in Colombia. The numerical results show an improve of $9.5 \%$ in the reliability with an increase of $9.8 \%$ on transportation total cost of the supply chain. However, in the proposed model, the companies would not have to incur adequacy costs of consolidation centers, given that current facilities have the capacity to become consolidation center without incurring on significant costs
\end{abstract}

Keywords: Reliability, disruptive event, supply chains, resilience optimization.

\footnotetext{
1 Ingeniería Industrial. Universidad Autónoma del Caribe. Barranquilla, Colombia. E-mail: fernando.gonzalez69@uac.edu.co; juan.escorcia@uac.edu.co; ingeniero.luispt@gmail.com

* Autor de correspondencia.
} 


\section{INTRODUCCIÓN}

En los últimos años la competencia a nivel empresarial ha aumentado debido a los efectos directos que trae la globalización de los mercados para las diferentes economías nacionales e internacionales.

Hoy no existen fronteras terrestres para las negociaciones entre empresas, lo que ha generado el aumento considerable de cadenas de suministro globales, trayendo como consecuencia una mayor exposición a eventos disruptivos, debido al alcance global de estas. Es decir, hoy día la probabilidad de falla de una cadena de suministro es mayor que en años anteriores, dado que las empresas actuales tienen mercados y proveedores más globalizados.

En los últimos años han ocurrido eventos que corroboran lo anterior: Tsunami en Indonesia (2004) y Japón (2011), el huracán Katrina (2005), el terremoto de Taiwán (1999, 2009 y 2010), el terremoto de Turquía (2012), el terremoto de Japón y Ecuador (2016), los ataques terroristas en París (2015), Nueva York (2001), Madrid (2004), Londres (2005), Yakarta (2009) y Mumbai (2008), las inundaciones en Tailandia (2011), en el sur del Atlántico, Colombia (2010), la recesión económica en Europa y USA, la variación súbita del dólar, entre otros.

Para que una cadena de suministro esté preparada ante la ocurrencia de eventos de tal magnitud, esta debe ser resiliente. En consecuencia, para que una cadena de suministro tenga tal característica es necesario tener en cuenta, al momento de realizar su diseño, además del factor económico, otros factores, ya que tenerlo en cuenta no es suficiente para tomar las mejores decisiones. En el presente documento se analiza el factor de confiabilidad, con el fin de determinar la localización óptima de las instalaciones que permitan minimizar la probabilidad de falla de la misma. La falta de estudios con respecto a la confiabilidad de las cadenas de suministro, dificulta que las empresas trabajen colectivamente para mitigar el impacto de los eventos disruptivos.

Antes de continuar, es necesario resaltar la diferencia que existe entre los conceptos de resiliencia, confiabilidad y el diseño de una cadena de suministro robusta. Según Ardila, Romero \& González [1], la confiabilidad, responde a condiciones conocidas del entorno, es decir, los riesgos a los que se tenga que enfrentar una cadena de suministro, los que son conocidos y asumidos por dicho sistema, sin que su desempeño se vea comprometido. Mientras que la robustez obedece a condiciones desconocidas del entono, para las que el sistema debe prever los riesgos e implementar estrategias que le permitan responder de mejor forma a la variabilidad inherente de dicho entorno. Por su parte, la resiliencia, a diferencia de la confiabilidad y la robustez del sistema, no busca estrategias que le permitan prevenir los riesgos de un posible evento disruptivo, sino, por el contrario, le deben permitir responder de la mejor forma posible, absorbiendo el impacto sobre el desempeño de las operaciones, y retornando de manera ágil al estado deseado de la cadena.

Teniendo en cuenta los conceptos anteriores, se puede decir que un modelo de fortificación de la cadena de suministro, aumentará la capacidad de distribución que tiene para absorber el impacto negativo originado por eventos disruptivos es decir, el diseño de una cadena de suministro teniendo en cuenta factores de confiabilidad, hace que el suministro sea más resiliente ante la ocurrencia de eventos disruptivos.

En este artículo se presenta el diseño de un modelo de programación no lineal entero mixto, para determinar la localización óptima y confiable de instalaciones en una cadena de suministro relacionada con empresas particulares del sector agroindustrial del Atlántico, Colombia.

Posterior al estado del arte se presenta el caso de estudio, el que especifica la situación actual de la cadena de suministro de la empresa Fruta Mundial, ubicada en el departamento del Atlántico, en Colombia. Seguidamente, se encuentra la modelación matemática del problema y se aprecian los resultados de las corridas del modelo. Finalmente, se presenta la discusión de los resultados, donde se evalúa el rendimiento del modelo diseñado teniendo en cuenta tres (3) enfoques distintos

\section{ESTADO DEL ARTE}

Para aclarar los conceptos de resiliencia y confiabilidad, a continuación se citan algunas investigaciones anteriores que han estudiado dichos conceptos aplicados a cadenas de suministros. 


\section{Resiliencia en cadenas de suministro}

Ponomarov \& Holcomb [2] definen la resiliencia en la cadena de suministros como la capacidad adaptativa de la cadena de prepararse para la ocurrencia de eventos inesperados, y recuperarse de ellos mediante el mantenimiento, la continuidad de las operaciones a un nivel deseado y el control sobre la estructura y funcionamiento

De acuerdo con Zobel [3], dos de las principales métricas que permiten la caracterización del concepto de resiliencia son el impacto inicial de la perturbación y el subsecuente tiempo de recuperación del sistema. Pero el gran problema con ellas, es que se trata de medidas posteriores a la ocurrencia de un evento disruptivo y difícilmente permiten su anticipación. Por lo que en su trabajo, Zobel [3] formuló un modelo de predicción multidimensional capaz de ajustar la función matemática que describe la resiliencia de un sistema a las preferencias y prioridades del tomador de decisiones.

En el trabajo desarrollado por Francis \& Bukera [4] se hace una revisión de los diferentes enfoques usados para definir el término de resiliencia y metodologías para su valoración. Esta revisión de distintos referentes, les permitió realizar la construcción de un marco para el análisis de la resiliencia partiendo de una clara identificación del sistema, los ajustes estructurales orientados a generar un sistema resiliente, el análisis de vulnerabilidad y el compromiso con los grupos de interés.

Con este marco, se pretende impulsar de desarrollo de tres características, o capacidades, distintivas de todo sistema resiliente: la capacidad de adaptación, de absorción y recuperación.

Diseño de cadenas de suministro bajo incertidumbre Una cadena de suministro es una configuración de instalaciones geográficamente ubicadas en pro de atender la demanda de una base de clientes [5]. El proceso de diseño de la red de cadenas de suministros envuelve decisiones como determinar el número, capacidad, y ubicación de las instalaciones de producción y distribución de las compañías pertenecientes [5]. Adicionalmente, en la mayoría de los casos se toman decisiones de nivel táctico, como estrategias de distribución, trasporte y manejo de inventarios y decisiones de nivel operativo como el cumplimiento de la demanda de los clientes [6].

Simchi-Levi \& Kaminsky [7] consideran que SCND es el paso primario y más importante para lograr un aumento en los beneficios de las redes de suministros, dado que las decisiones que se tomen en este paso tienen un impacto importante sobre el retorno de la inversión de la cadena, por tanto el objetivo de SCND es diseñar una estructura de red eficiente para las entidades pertenecientes a una nueva cadena o rediseñar una red existente para aumentar su valor total. En este paso, se toman decisiones como el número de niveles de la cadena, la ubicación y capacidad de las instalaciones en cada nivel y el flujo de material / producto en toda la red.

Cuando se realiza un horizonte de planeación a largo plazo se hace necesario considerar un entorno dinámico y estocástico, por tanto, no es suficiente solo considerar variables aleatorias como demanda, precios, tasas de cambio, si no que se deben incluir eventos extremos como desastres naturales, ataques terroristas que pueden seriamente afectar la capacidad y operación de la red de suministros [5]. Tradicionalmente enfoques para el diseño de redes de cadenas de suministros asumen entornos determinísticos, lo que da lugar a modelos de ubicación-asignación clásicos [8].

Algunas extensiones de los modelos clásicos determinísticos tienen en cuenta factores aleatorios mediante programación estocástica [9] o tienen en cuenta fallas de depósito utilizando optimización robusta [10]. Sin embargo, se presenta en la literatura una carencia de investigación en SCND considerando tantos factores aleatorios usuales del negocio e interrupciones de alto impacto [5].

En cuanto al objetivo en consideración: la incertidumbre, se debe tener en cuenta el riesgo existente en el momento de la toma de decisiones. Una definición general de riesgo es la probabilidad de un daño o pérdida u ocurrencia de eventos negativos. Para reducir los efectos negativos de los riesgos inherentes, se deben aplicar algunas acciones como identificación, evaluación, priorización, seguimiento y control.

Según Chopra \& Sodhi [11], diferentes tipos de riesgos se encuentran asociados a las cadenas de 
suministros, entre los que se encuentran por ejemplo las demoras, pronósticos errados, fracasos en la contratación, interrupción de operaciones, averías de los sistemas, infracciones de la propiedad intelectual, problemas con la capacidad y el inventario. Cualquiera de estos amenaza el rendimiento global de las SC y su rentabilidad, por lo tanto, la gestión de riesgos es una preocupación importante de la gestión de la cadena de suministro y podrían gestionarse mediante el diseño de una red sólida y una gestión eficiente del flujo del producto [6].

Investigaciones que abordan el diseño integral de redes de cadenas de suministro en condiciones de incertidumbre son significativamente menores en número a las realizadas en condiciones determinísticas. Gutiérrez, Kouvelis \& Kurawala [12], proponen un marco de optimización robusta para el diseño de red en condiciones de incertidumbre. Este enfoque busca configuraciones de red que son buenas (casi óptimas) para una variedad de escenarios. Los autores proponen una modificación del algoritmo de descomposición de Benders, que se utiliza comúnmente para problemas determinísticos de diseño de la red.

Para reducir los efectos negativos de las perturbaciones en la cadena de suministros, y hacerla más resistente, se deben implementar estrategias de mitigación, aunque en ocasiones esto lleva a situaciones en las que se debe cambiar gran parte del sistema de distribución, por lo tanto, es importante ensayar estas estrategias mediante un modelo de simulación y evaluar los efectos que se puedan presentar [13].

Para la solución de modelos de programación se utiliza la simulación el que es un proceso que se enfoca en la optimización, este es un sistema, que es representado por un modelo de simulación, y se ejecuta para un determinado período de tiempo. El rendimiento del sistema se basa en una salida del modelo de simulación y la respuesta para ser optimizado [14].

En el documento [15] consideran un modelo de dos etapas para un multiperíodo de planificación de la capacidad de las redes de la cadena de suministro, en el que las primeras decisiones de la etapa se realizarán antes de considerar futuras demandas, en temas relacionados a las aperturas y cierres de plantas y centros de distribución, y la fijación de sus niveles de capacidad. Luego, basándose en el escenario de demanda particular, se realizan las decisiones de producción y distribución de manera óptima. El objetivo general es minimizar el costo de las decisiones estratégicas de la primera etapa y los costos de producción y distribución que se espera en los escenarios de demanda incierta. Se utilizó descomposición de Benders para resolver el problema estocástico resultante.

El mismo tipo de enfoque también fue propuesto por Pan \& Nagi [16], que proponían una red múltiple de la cadena de suministro, considerando principalmente decisiones de distribución, producción, inventario y ubicación de las instalaciones.

Tsiakis, Shah \& Pantelides [17], consideran un modelo de programación estocástica de dos etapas para el diseño de red de la cadena de suministro bajo demanda incertidumbre. Los autores desarrollaron un modelo de programación lineal entera mixta a gran escala de este problema, y presentaron un caso estudio utilizando una cadena de suministro europea que incluye a múltiples productos, ubicaciones de los clientes, ubicaciones de los centros de distribución, escenarios de demanda.

Nickel, Saldanha-da-Gama \& Ziegler [18], consideran un problema multiperíodo proponiendo varios productos en diseño de la red de la cadena de suministro con decisiones financieras y de gestión de riesgos bajo incertidumbre. Considerando un horizonte de planeación dividido en varios períodos de tiempo, en los que se deben tomar varias decisiones:

1. Las instalaciones que deben estar operando $\mathrm{y}$, por lo tanto, las inversiones a realizar en la estructura de la cadena de suministro.

2. Otras inversiones para hacer además de los anteriores.

3. Los préstamos a obtener.

4. El flujo de mercancías por medio de la red.

Un conjunto de escenarios se proponen para describir la incertidumbre, los ingresos se incluyen en el modelo así como el retorno de la inversión. El nivel de servicio para cada cliente se evalúa y pondera en la función objetivo; por último, se introduce una medida de riesgo. El objetivo es minimizar el costo total que se evalúa teniendo en cuenta las inversiones realizadas, los ingresos y los costos 
de transporte. El problema se formula como un problema de programación estocástica multietapa, entero mixto [18].

Santoso, Ahmed, Goetschalckx \& Shapiro [19], integran una estrategia de muestreo propuesta recientemente, muestreo aproximación media (SAA), con un algoritmo de descomposición de Benders acelerado para resolver los problemas de diseño de la cadena de suministro con distribuciones continuas de los parámetros inciertos, por lo tanto un número infinito de escenarios. La técnica algorítmica propuesta es inmediatamente aplicable a problemas con finita pero un número extremadamente grande de escenarios. La metodología propuesta puede servir como una herramienta de planificación estratégica viable, lo que permite a los planificadores investigar una multitud de posibilidades y una variedad de soluciones.

Finalmente, Santander, Amaya, Viloria, Sierra, Saavedra, Romero \& González [20] desarrollan modelos de optimización donde se relacionan dos objetivos al momento de diseñar la cadena de suministro, costos y confiabilidad. En su investigación despliegan una relación de costos/confiabilidad para lograr un equilibrio entre ambas soluciones.

La confiabilidad está dada por $S^{A} \times S^{N}$, donde $S^{A}$ es la confiabilidad de los arcos y $S^{N}$ representa la confiabilidad de los nodos.

$$
\begin{aligned}
& S^{A}=\prod_{\forall i \in I} \prod_{\forall j \in J}\left[\left(S_{i j}^{I J}\right)^{B_{i j}^{I J}}\right] \times \prod_{\forall j \in J} \prod_{\forall k \in K}\left[\left(S_{j k}^{I K}\right)^{B_{i j k}^{I K}}\right] \\
& \times \prod_{\forall k \in K} \prod_{\forall m \in M}\left[\left(S_{k m}^{K M}\right)^{B_{k m}^{K M}}\right] \\
& S^{N}=\left(\prod_{\forall i \in I}\left(S_{i}^{I}\right)^{Y_{i}^{I}}\right) \times\left(\prod_{\forall j \in J}\left(S_{j}^{J}\right)^{Y_{j}^{J}}\right) \times\left(\prod_{\forall k \in K}\left(S_{k}^{K}\right)^{Y_{k}^{K}}\right)
\end{aligned}
$$

Teniendo en cuenta la revisión literaria realizada, se puede decir que los modelos para seleccionar la localización de instalaciones se encuentran limitados en la mayoría de los casos a la interacción de un solo criterio, ya sea el costo, distancia del punto a los clientes o cobertura de demanda. Se encontró un solo trabajo donde se aplican objetivos múltiples relacionados con costos y confiabilidad, pero cuya complejidad matemática de solución es alta debido a las relaciones que presentan sus variables.

Por tal motivo, en esta investigación se presenta un modelo matemático con una función objetivo diferente a las presentadas en las investigaciones consultadas, donde se logren funciones de confiabilidad con relaciones matemáticas menos complejas. El modelo de este proyecto tiene el objetivo de minimizar la división del costo total sobre la confiabilidad, esto matemáticamente representa minimizar los costos y maximizar la confiabilidad. Esto permite que la solución de este modelo seleccione las localizaciones de las instalaciones óptimas bajo ambos criterios.

\section{CASO DE ESTUDIO}

\section{Situación actual de la cadena de suministro}

El caso de estudio se desarrolló para un segmento de la cadena de suministro de una empresa perteneciente al sector agroindustrial en la región norte de Colombia. Esta se encuentra conformada por dos eslabones; el eslabón 2 está conformado por 19 fincas, clasificadas en 12 fincas pequeñas (agrupadas en 3 asociaciones) y 7 fincas grandes, encargados de la cosecha y suministros del producto en cuestión.

En la Figura 1 se observa la estructura actual de la red de suministros de Fruta Mundial, en donde se pueden observar las tres asociaciones conformadas por las fincas pequeñas.

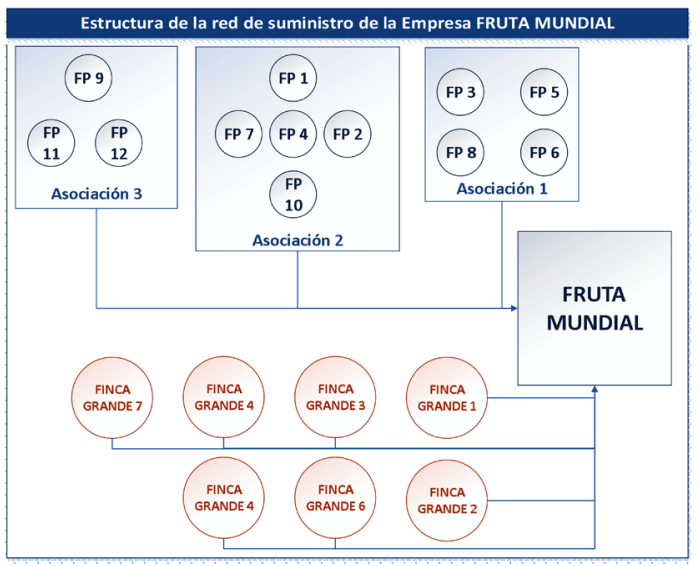

Figura 1. Estructura actual de la red suministro de Fruta Mundial. 
Las diferencias entre los dos tipos de fincas radican en la cantidad ofertada, donde las fincas pequeñas presentan una oferta promedio semanal de 30 toneladas y las fincas grandes una oferta promedio semanal de 574 toneladas. Otra diferencia es el agrupamiento en asociaciones por parte de las fincas pequeñas. Las fincas son encargadas de la cosecha y suministros del producto en cuestión. El eslabón 1 se encuentra constituido por una fábrica la que es encargada de transformar el producto y despachos a clientes externos.

La cadena de suministro de la empresa trabaja según la disponibilidad de materia prima, para lo que cuenta con una planta principal ubicada en la ciudad de Barranquilla y cuyos proveedores, principalmente, están distribuidos en toda la región Caribe. Algunos de los proveedores cuentan con centros donde preparan grandes cantidades de mangos para ser enviados mientras otros los envían directamente desde las fincas donde se recolecta la materia prima.

La mayor parte de la materia prima de la empresa es recibida en la empresa en los meses de abril a julio, desde sus proveedores que están ubicados en gran parte de la región Caribe de Colombia.

En la etapa inicial de la investigación de detectó que el principal problema presentado en la cadena de suministro es que la empresa productora no cuenta con una buena programación para recepción de materia prima, y esto genera congestiones de más de 100 camiones de diferentes capacidades en fila, quienes quedan a la espera de que la materia prima sea recibida por la empresa. Debido al largo tiempo de espera en los que incurren estos camiones se genera un incremento en las unidades no conformes.

Asimismo, los daños en las carreteras, cierres, embotellamientos, fenómenos naturales, entre otros, son algunos de los factores que dificultan el tiempo de envío de materia prima del proveedor a la empresa. Por este motivo, el contar con varias rutas alternas, ayudará a evitar que problemas en una ruta afecten el suministro de materia prima hacia la empresa.

Para coordinar los envíos de materia prima de tal forma que se logre minimizar el impacto generado por las filas presentadas en la fábrica, se pretende crear unos centros de consolidación para las fincas pequeñas y fincas grandes. Estos centros de consolidación recolectarán la materia prima de los diferentes proveedores y posteriormente se enviaran a la fábrica.

Se espera disminuir el número de camiones que llegan a la empresa productora, y establecer un punto óptimo de recolección para las fincas.

La red de suministros trabaja con cinco tipos de camiones donde su capacidad se encuentra dada en toneladas: (1) 3 ton, (2) 5 ton, (3) 10 ton, (4) 18 ton y (5) 30 ton. Dadas sus políticas de compra, Fruta Mundial semanalmente se abastece del total de la capacidad ofrecida por sus proveedor que es, en promedio, 4.023 toneladas/semanales.

Finalmente, se establecen los datos de la cadena actual y se procede a resolver el modelo, razón por la que se buscan determinar los costos de transportes y la confiabilidad de la cadena de suministro actual. Los costos de las rutas son calculados teniendo en cuenta la distancia entre estos. Las probabilidades de funcionamiento y la capacidad de los centros de consolidación son supuestas por los autores de manera aleatoria.

\section{MODELACIÓN MATEMÁTICA}

Para determinar las localizaciones óptimas de los centros de consolidación, se elabora un modelo de programación no lineal entero, con el objetivo de minimizar los costos totales en que se incurren, tanto en el transporte como en la instalación de los mismos y maximizar la probabilidad de que toda la cadena no sea afectada por ningún tipo de falla en ninguno de sus eslabones y arcos de transporte.

También se trata de buscar que la cadena que sea seleccionada como la solución del modelo, cumpla con un alto nivel de confiabilidad. El siguiente modelo es descrito de forma general, para facilitar el uso en posteriores estudios:

\section{Conjuntos}

$I$ = Conjunto de fincas tipo $i$.

$J$ = Conjunto de centros de consolidación $(C C)$ tipo $j$.

$M=$ Conjunto de camiones tipo $m$. 


\section{Parámetros}

$n \quad=$ Número total de fincas.

$w \quad=$ Número total de posibles $C C$.

$r \quad=$ Número total de tipos de camiones.

$C_{i j m}^{F} \quad=$ Costo de transportar la mercancía desde la finca tipo $i$ al $C C$ tipo $j$ por medio del camión tipo $m$ (este es el costo de transportar la capacidad total de una camión).

$C_{j m}^{C C} \quad=$ Costo de transportar la mercancía desde el $C C$ tipo $j$ a la fábrica por medio del camión tipo $m$.

$A_{j} \quad=$ Costo de la apertura y funcionamiento del $C C$ tipo $j$.

$K_{m}^{C} \quad=$ Capacidad en toneladas del camión tipo $m$.

$K_{i}^{F} \quad=$ Capacidad de producción en toneladas de la finca tipo $i$.

$K_{j}^{C C}=$ Capacidad en toneladas del $C C$ tipo $j$.

$P_{i}^{F} \quad=$ Probabilidad de que la finca tipo $i$ funcione.

$P_{j}^{C C} \quad=$ Probabilidad de que el $C C$ tipo $j$ funcione.

$P_{i j}^{F-C C}=$ Probabilidad de que la ruta de la finca tipo $i$ al $C C$ tipo $j$ funcione.

$P_{j}^{C C-F A B}=$ Probabilidad de que el arco del $C C$ tipo $j$ a la fábrica funcione.

$P^{F A B}=$ Probabilidad de que la fábrica funcione.

$D^{F A B}=$ Demanda anual de la fábrica en toneladas.

\section{Variables}

$X_{i j}^{F} \quad=$ Cantidad de toneladas a transportar de la finca tipo $i$ al $C C$ tipo $j$.

$X_{j}^{C C} \quad=$ Cantidad de toneladas a transportar del $C C$ tipo $j$ a la fábrica.

$Y_{j} \quad=$ Variable binaria que indica si se da apertura al $C C$ tipo $j$.

$L_{i j} \quad=$ Variable binaria que indica si la finca tipo $i$ envía al $C C$ tipo $j$.

$H_{i j m}^{F} \quad=$ Cantidad de envíos en camiones tipo $m$ utilizados de la finca tipo $i$ para transportar toneladas al $C C$ tipo $j$.

$H_{j m}^{C C} \quad=$ Cantidad de envíos en camiones tipo $m$ utilizados por el $C C$ tipo $j$ para enviar toneladas a la fábrica.

\section{Función Objetivo}

La función objetivo se divide en dos partes: la primera, es la parte de los costos en los que incurre el modelo y, la segunda, es la parte de la confiabilidad que genera la solución del modelo:

\section{$1^{\circ}$ Parte. Costos:}

Se tiene en cuenta los costos de apertura del centro de consolidación más los de transporte de las fincas a los centros de consolidación y de los centros de consolidación a la fábrica.

$$
\begin{aligned}
\text { Costos }= & \sum_{j=1}^{w} A_{j} Y_{j}+\sum_{i=1}^{n} \sum_{j=1}^{w} \sum_{m=1}^{r} H_{i j m}^{F} C_{i j m}^{F}+ \\
& \sum_{j=i}^{w} \sum_{m=1}^{r} H_{j m}^{C C} C_{j m}^{C C}
\end{aligned}
$$

\section{$2^{o}$ Parte. Confiabilidad:}

Se supone que todos los eventos son independientes, la probabilidad de que funcione cada ruta (finca- $C C$ fabrica) está dada por la siguiente ecuación, donde se multiplican la probabilidad de que funcione la finca, el $C C$ y cada uno de los transporte de la ruta por la variable binaria que determina si la finca le envía al $C C$ :

$$
\begin{aligned}
& \text { Confiabilidad } \text { Ruta }_{i j}= \\
& P_{i}^{F} P_{j}^{C C} P_{i j}^{F-C C} P_{j}^{C C-F A B} P^{F A B} L_{i j}
\end{aligned}
$$

La probabilidad de que todas las rutas de la cadena de suministro funcionen es la productoria de cada ruta habilitada de la cadena; se define como, $P^{T}$, esta indica la probabilidad de funcionamiento de todo el sistema, como se muestra a continuación:

$$
\begin{aligned}
P^{T}= & \prod_{i=1 \forall L i j=1}^{n} \prod_{j=1 ; \forall L i j=1}^{w} \\
& \left(P_{i}^{F} P_{j}^{C C} P_{i j}^{F-C C} P_{j}^{C C-F A B} P^{F A B} L_{i j}\right)
\end{aligned}
$$

Para la solución del modelo en GAMS es complejo restringir que solo se aplique la productoria a las rutas abiertas; por tal motivo, se diseñó la siguiente ecuación la que trabaja con las probabilidad de no funcionamiento de cada una de las rutas y las multiplica por la variable de apertura de ruta, luego a 1 se le resta este valor, de tal forma que las rutas no habilitadas en vez de 0 den 1 y pueda hacerse la productoria sin afectar la probabilidad de funcionamiento de la cadena:

$$
\begin{aligned}
P^{T}= & \prod_{i=1}^{n} \prod_{j=1}^{w}\left(1-\left(1-P_{i}^{F}\right) * L_{i j}\right) *\left(1-\left(1-P_{j}^{C C}\right) * L_{i j}\right) \\
& *\left(1-\left(1-P_{i j}^{F-C C}\right) * L_{i j}\right) \\
& *\left(1-\left(1-P_{j}^{C C-F A B}\right) * L_{i j}\right) \\
& *\left(1-\left(1-P^{F A B}\right) * L_{i j}\right)
\end{aligned}
$$


La función objetivo total queda dada por la división entre los costos y la probabilidad de funcionamiento de la cadena de suministro. Al realizar esta relación se logra que el modelo al seleccionar las rutas óptimas considere los costos y la confiabilidad de la misma. Por ejemplo, supóngase la ruta A y B que tienen un coste de 100 y 95 , respectivamente, la ruta A tiene una confiabilidad del $90 \%$ mientras la ruta $\mathrm{B}$ del $70 \%$. Al dividir costos/confiabilidad se escoge la ruta A dado que minimiza la función objetivo. Por tanto, la relación propuesta asegura la selección teniendo en cuenta tanto los costos como la confiabilidad de la ruta.

$$
Z_{\min }=\frac{\text { Costos }}{P^{T}}
$$

La relación anterior es estudiada en el trabajo de Santander, Amaya, Viloria, Sierra, Saavedra, Romero, González [20].

\section{Restricciones}

1. Restricción de cantidad enviada de cada $C C$ a la empresa: La sumatoria de las cantidades enviadas de cada una de las fincas al $C C$ debe ser igual a la cantidad enviada del $C C$ a la fábrica, esto aplicado a cada $C C$.

$$
\sum_{i=1}^{n} X_{i j}^{F}=X_{j}^{C C} ; \forall j \in J
$$

2. Restricción de cantidad enviada desde los $C C$ a la fábrica: La sumatoria de las cantidades enviadas de cada $C C$ a la fábrica tienen que ser igual a la demanda total de la fábrica (en una cadena balanceada).

$$
\sum_{j=1}^{w} X_{j}^{C C}=D^{F A B}
$$

3. Restricción de capacidad de los camiones de las fincas a los $C C$ : La cantidad de envíos del camión tipo $m$ de la finca por la capacidad de dichos camiones, multiplicado por la variable de apertura de la ruta, da como resultado la capacidad por tipo de camión, a lo que se suma por la de cada uno de los diferentes tipos de camiones, tiene que ser mayor o igual a las cantidades enviadas de la finca al $C C$, lo que debe realizarlo para cada una de las fincas y cada $C C$. Se busca que la capacidad conjunta de todos los camiones sea mayor que la cantidad que la finca puede enviar, siendo un objetivo para cada finca.

$$
\sum_{m-1}^{r} L_{i j} H_{i j m}^{F} K_{m}^{C} \geq X_{i j}^{F} ; \forall i \in I^{\wedge} \forall j \in J
$$

4. Restricción de capacidad de los camiones de los $C C$ a la fábrica: La cantidad de envíos del camión tipo $m$ del $C C$ a la fábrica multiplicado por la capacidad de dichos camiones y la variable de apertura del $C C$, sumado por la de cada uno de los diferentes tipo de camiones, tiene que ser mayor o igual a las cantidades enviadas del $C C$ a la fábrica. Lo anterior, debe realizarse para cada una de los $C C$.

$$
\sum_{m=1}^{r} H_{j m}^{C C} K_{m}^{C} Y_{j} \geq X_{j}^{C C} ; \forall j \in J
$$

5. Restricción de variable binaria: La sumatoria de la variable binaria de si la finca envía toneladas al $C C$, tiene que ser igual a 1 para que solo exista una relación entre una finca y un $C C$, lo que se aplica para todas las fincas.

$$
\sum_{j=1}^{m} L_{i j}=1 ; \forall i \in I
$$

6. Restricción de capacidad de los centros de consolidación: La sumatoria de las toneladas enviadas desde las diferentes fincas a un $C C$, tiene que ser menor o igual que la capacidad del $C C$ multiplicado por el variable si se abre el $C C$ o no. Lo anterior, aplica para todos los $C C$.

$$
\sum_{i=1}^{n} X_{i j}^{F} \leq K_{j}^{C C} Y_{j} ; \forall j \in J
$$

7. Restricción de las fincas: La sumatoria de las toneladas enviadas de la finca a los diferentes $C C$ tiene que ser menor o igual que la capacidad de la finca. Esta acción se emplea para cada una de las fincas.

$$
\sum_{j=1}^{w} X_{i j}^{F} \leq K_{i}^{F} ; \forall i \in I
$$


8. Restricciones de apertura de la ruta entre finca y $C C$ : La variable de si la finca envía al $C C$ debe ser menor o igual la variable si se abre el $C C$ para cada tipo de finca y cada tipo de $C C$. Su objetivo es el de una finca solo envíe a los $C C$ abiertos.

$$
L_{i j} \leq Y_{j} ; \forall i \in I^{\wedge} \forall j \in J
$$

9. Restricciones de variables binarias: Se limita a los números 0 y 1 las variables de apertura de $C C$ y la de apertura e ruta de la finca al $C C$, para cada finca y cada $C C$.

$$
Y_{j}, L_{i j}=\{0,1\} ; \forall i \in I^{\wedge} \forall j \in J
$$

10. La probabilidad de funcionamiento de cada ruta: Se describió en la sección de la función objetivo, en la ecuación (2).

$P_{i j}=P_{i}^{F} P_{j}^{C C} P_{i j}^{F-C C} P_{j}^{C C-F A B} P^{F A B} L_{i j} ; \forall i \in I^{\wedge} \forall j \in J$

11. Probabilidad de funcionamiento total de la cadena: Se describió en la sección de la función objetivo, en la ecuación (3).

$$
P^{T}=\prod_{i=1}^{n} \prod_{j=1 ;}^{w}\left(\begin{array}{l}
\left(1-\left(1-P_{i}^{F}\right) * L_{i j}\right) \\
*\left(1-\left(1-P_{j}^{C C}\right) * L_{i j}\right) \\
*\left(1-\left(1-P_{i j}^{F-C C}\right) * L_{i j}\right) \\
\left.*\left(1-P_{j}^{C C-F A B}\right) * L_{i j}\right) \\
*\left(1-\left(1-P^{F A B}\right) * L_{i j}\right)
\end{array}\right)
$$

12. Restricción de no negatividad: Se limita a solo números positivos todas las variables del problema.

$$
\begin{gathered}
X_{i j}^{F}, X_{j}^{C C}, Y_{j}, L_{i j}, H_{i j m}^{F}, H_{j m}^{C C}, P^{T} \geq 0 ; \forall \\
h i \in I^{\wedge} \forall j \in J^{\wedge} \forall m \in M
\end{gathered}
$$

\section{RESULTADOS DEL MODELO ACTUAL}

En esta sección se encuentran los resultados de la situación actual estudiada, en la que tres centros de consolidación solo están habilitados para las fincas pequeñas, mientras las fincas grandes envían directamente a la fábrica. Para solucionar el modelo planteado con sus diferentes escenarios se utilizó solver Lindo en el software Gamside versión 24.5.4.

En cuanto a los resultados arrojados por el software se encuentran:

- Toneladas enviadas desde cada finca a cada $C C$ o directo a la fábrica.

- Número de viajes por tipo de camión desde cada finca hacia los centros de consolidación y Fruta Mundial.

- Número de viajes por tipo de camión desde los puntos de consolidación hacia Fruta Mundial.

- Los costos de transporte asociados a la situación actual.

En la Tabla 1 se presentan las toneladas enviadas desde las diferentes fincas a los centros de consolidación o Fruta Mundial, dependiendo del tamaño de la finca, ya que las pequeñas envían a los centros de consolidación y las grandes directo a la fábrica.

Tabla 1. Toneladas enviadas desde las fincas hacia los puntos de consolidación y Fruta Mundial.

\begin{tabular}{|c|c|c|}
\hline Finca & $\boldsymbol{C C}$ & Toneladas \\
\hline 1 & 1 & 32 \\
\hline 2 & 1 & 32 \\
\hline 3 & 2 & 49 \\
\hline 4 & 1 & 32 \\
\hline 5 & 2 & 19 \\
\hline 6 & 2 & 31 \\
\hline 7 & 1 & 27 \\
\hline 8 & 2 & 13 \\
\hline 9 & 3 & 36 \\
\hline 10 & 1 & 23 \\
\hline 11 & 3 & 31 \\
\hline 12 & 3 & 29 \\
\hline 13 & Directo fab. & 438 \\
\hline 14 & Directo fab. & 500 \\
\hline 15 & Directo fab. & 594 \\
\hline 16 & Directo fab. & 725 \\
\hline 17 & Directo fab. & 529 \\
\hline 18 & Directo fab. & 617 \\
\hline 19 & Directo fab. & 620 \\
\hline
\end{tabular}

En la Tabla 2 se presentan el número de viajes necesarios por tipo de camión para el trasporte, según los resultados de la Tabla 1. 
Tabla 2. Número de viajes por tipo de camión desde las fincas pequeñas a los centros de consolidación y de las fincas grandes directo a Fruta Mundial.

\begin{tabular}{|c|c|c|c|c|c|}
\hline Finca & Camión & \# de Viajes & Finca & Camión & \# de viajes \\
\hline 1 & 6 & 1 & \multirow{2}{*}{11} & 1 & 1 \\
\hline \multirow{2}{*}{2} & 1 & 1 & & 5 & 1 \\
\hline & 5 & 1 & 12 & 5 & 1 \\
\hline 3 & 6 & 1 & \multirow{2}{*}{13} & 4 & 1 \\
\hline \multirow{2}{*}{4} & 1 & 1 & & 5 & 14 \\
\hline & 5 & 1 & 14 & 5 & 17 \\
\hline 5 & 5 & 1 & 15 & 5 & 20 \\
\hline \multirow{2}{*}{6} & 1 & 1 & \multirow{2}{*}{16} & 2 & 1 \\
\hline & 5 & 1 & & 5 & 24 \\
\hline 7 & 5 & 1 & 17 & 5 & 18 \\
\hline 8 & 4 & 1 & \multirow{2}{*}{18} & 4 & 1 \\
\hline 9 & 6 & 1 & & 5 & 20 \\
\hline 10 & 5 & 1 & 19 & 5 & 21 \\
\hline
\end{tabular}

En la Tabla 3 se muestran la cantidad de viajes necesarios por tipo de camión, para el flujo de la mercancía desde los puntos de consolidación hasta la fábrica.

Tabla 3. Número de viajes por tipo de camión desde los puntos de consolidación hacia Fruta Mundial.

\begin{tabular}{|c|c|c|}
\hline $\boldsymbol{C} C$ & Tipo camión & Numero viajes a Fruta Mundial \\
\hline 1 & 5 & 5 \\
\hline 2 & 5 & 4 \\
\hline \multirow{2}{*}{3} & 1 & 1 \\
\cline { 2 - 3 } & 5 & 3 \\
\hline
\end{tabular}

De acuerdo con los resultados anteriores para la distribución de 4.377 toneladas de producto, se tienen unos costos semanales totales de \$280.001.000 COP (96.552 USD) y la fábrica estaría recibiendo 150 camiones semanalmente. El valor de la confiabilidad de la cadena de suministro actual es $17,7 \%$, lo que se desglosa de la confiabilidad de las rutas que intervienen en cada uno de los centros de consolidación.

\section{APLICACIÓN DEL MODELO PROPUESTO}

Dado, que en la red de suministro estudiada se presentan problemas en la coordinación del envío de materia prima a la fábrica, lo que genera costos asociados a los daños de la materia prima, debido al tiempo que tienen que esperar los camiones para ser recibidos por la empresa (Fabrica), se propone diseñar unos centros de consolidación para todas las fincas, de forma que todas ellas envíen a los centros de consolidación y que se aumente la confiabilidad de funcionamiento, teniendo un mínimo incremento en los costos, para que, de este modo, tener una integración efectiva con los proveedores y la empresa Fruta Mundial.

Es importante resaltar que la red de suministro en cuestión, no tiene en su planificación incurrir en costos de infraestructura para la creación de los probables centros de consolidación. Por tanto, se establecen como posibles puntos de consolidación las fincas grandes, y solo se incurrirá en costos de adecuación para que las fincas seleccionadas puedan recibir productos de otras fincas.

Para determinar las localizaciones óptimas de los centros de consolidación bajo los criterios de costos y confiabilidad, se aplicará el modelo de programación no lineal entero, el que tiene como objetivo minimizar la división de los costos totales en que se incurren, tanto los transportes como los de adecuación, dividido por la confiabilidad de la cadena, como se presenta en la ecuación (5). Lo anterior, debido a que se busca que la cadena seleccionada como la solución, cumpla con un alto nivel de confiabilidad para minimizar el impacto de los eventos disruptivos (daños en las carreteras, cierres, colapso en la vía, fenómenos naturales, entre otros). 
De esta manera, se pretende aumentar la confiabilidad de la cadena de suministro, es decir, aumentar la capacidad de absorber el impacto negativo generado por la ocurrencia de un evento disruptivo, aunque este aumento genere en un incremento en los costos totales.

\section{RESULTADOS DEL MODELO PROPUESTO}

Para examinar el comportamiento del modelo, se plantean tres enfoques donde se cambia la función objetivo. El primer escenario es minimizar la relación costos sobre confiabilidad, el segundo es minimizar costos y el tercero maximizar la confiabilidad. A continuación, se puede apreciar la configuración para cada uno de los casos; los rectángulos representan los centros de consolidación.

En la Figura 2 se presenta la cadena de suministro bajo el primer escenario (minimizar costos sobre confiabilidad); los centros de consolidación deben establecerse en la finca grande 4 y 6 .

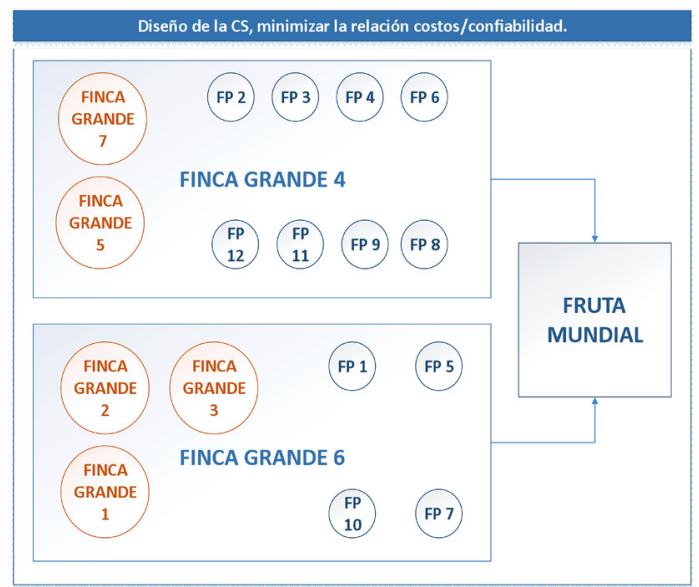

Figura 2. Diseño de la CS, minimizar la relación costos/confiabilidad.

En la Figura 3 se presenta la cadena de suministro bajo el segundo escenario (minimizar costos), los centros de consolidación deben establecerse en la finca grande 4 y 6 .

En la Figura 4 se presenta la cadena de suministro bajo el tercer escenario (maximizar confiabilidad), los centros de consolidación deben establecerse en la finca grande 1,4 y 7 .

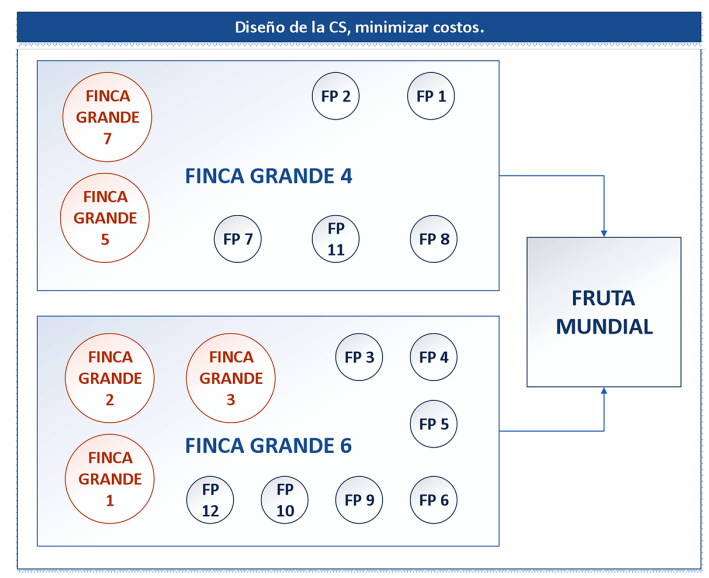

Figura 3. Diseño de la CS, minimizar costos.

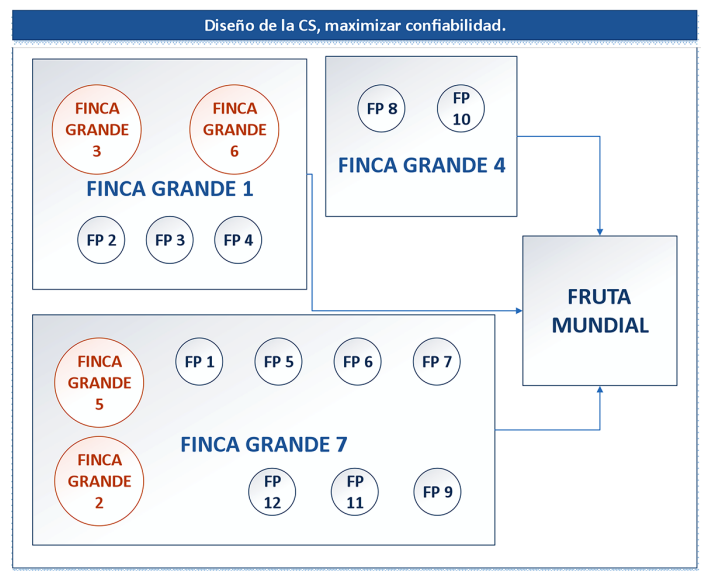

Figura 4. Diseño de la CS, maximizar confiabilidad.

Los valores de confiabilidad presentan un incremento del $9,5 \%, 7,5 \%$ y $23,7 \%$, respectivamente, con respecto al estado actual, esto puede verse en la Figura 5.

Relación costos de transportey confiabilidad

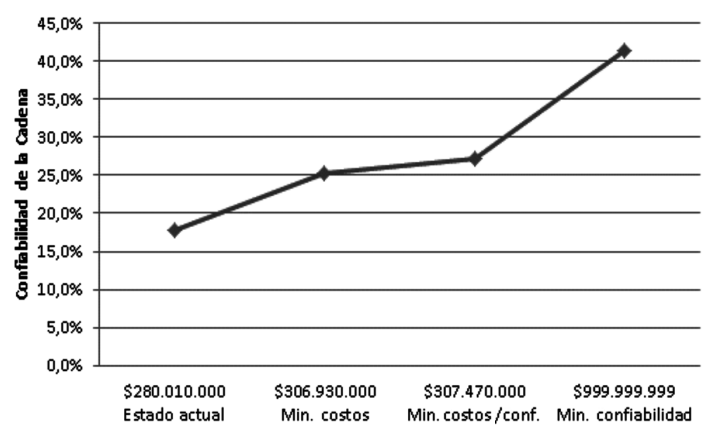

Figura 5. Relación costo de transporte y confiabilidad. 
La máxima confiabilidad que puede alcanzar la cadena teniendo en cuenta las condiciones del estado propuesto es de $41,4 \%$, la que se logra teniendo el objetivo de maximizarla sin importar en los costos que la cadena incurre.

La confiabilidad de la cadena actual es menor a las tres soluciones propuestas. Otro factor importante son los costos de transporte, los que se determinan restándole a los costos totales el costo de apertura del centro de consolidación por la cantidad de centros de consolidación que se abren.

Se aprecia un incremento después de deducir los costos de apertura de los centros de consolidación para cada caso de \$27.460.000 COP (9.468 USD), \$26.920.000 COP (9.282 USD) y \$ 147.099.019.990.000 COP (50.723.799.996 USD), respectivamente con respecto al estado actual. Esto se puede ver en la Figura 5.

Los costos asociados al escenario de maximizar la confiabilidad no son representativos, ello debido a que el modelo no restringe las decisiones de las variables que tienen costos asociados, motivo el cual toman valores extremadamente altos.

Un factor importante que se estudia son las cantidades totales de camiones que llegan a la fábrica cada semana, para cada uno de los escenarios propuestos y el estado actual. Ello se aprecia en la Tabla 4.

Tabla 4. Comparación de las cantidades de camiones que se envían a la fábrica para cada escenario.

\begin{tabular}{|l|c|c|c|}
\hline & $\begin{array}{c}\text { Tipo de } \\
\text { camión }\end{array}$ & Cantidad & Total \\
\hline \multirow{2}{*}{ Estado actual } & 2 & 1 & \multirow{2}{*}{150} \\
\cline { 2 - 3 } & 4 & 4 & \\
\cline { 2 - 3 } & 5 & 145 & \multirow{2}{*}{146} \\
\hline $\begin{array}{l}\text { Relación costo/ } \\
\text { confiabilidad }\end{array}$ & 5 & 146 & 146 \\
\hline Costos & 6 & 146 & \\
\hline
\end{tabular}

Se tiene una disminución de cuatro camiones menos semanalmente para los dos primeros escenarios, que anualmente representa a la empresa Fruta Mundial 208 envíos menos. No se tiene en cuenta la solución del escenario de maximizar la confiabilidad debido a que en este no se restringe las cantidades de los camiones.

\section{CONCLUSIONES}

La presente investigación abarcó el diseño de cadenas de suministros teniendo en cuenta dos objetivos fundamentales como son la disminución de los costos de la cadena de suministro y aumentar la confiabilidad de la misma, para lo que se diseñó un modelo de programación no lineal entero. Se llevó a cabo la experimentación de los resultados tomados como base una cadena de suministro del sector agroindustrial colombiano.

Como se mencionó anteriormente uno de los fenómenos observados en la red de suministros es la falta de coordinación entre las cantidades ofertadas por las fincas y las cantidades demandas por la fábrica (Fruta Mundial).

Dicha falta de coordinación ocasiona la pérdida de materias primas debido a su corto ciclo de vida, y como se demuestra en la presente investigación al modelar diferentes casos, se aumenta la confiabilidad teniendo un incremento de $9,8 \%$ y $9,6 \%$ para los enfoques 1 y 2 .

En estado actual de la cadena de suministro presenta una confiabilidad de $17,7 \%$, la que puede aumentarse a $27,2 \%$, adecuando la cadena de suministro a los resultados del escenario minimizar costos/confiabilidad, incrementarían el costo de transporte en \$27.460.000 COP (9469 USD) $(9,8 \%)$ con respecto a los costos totales, además de incurrir en los costos de adecuación de los centros de consolidación ubicados en las fincas 16 (Santo Tomás) y 18 (Caracolí).

El diseño de estos centros de consolidación, aparte de aumentar la confiabilidad de la cadena de suministro, le permitirá a la empresa (fábrica), poder coordinar el envío de la mercancía de estos centros de consolidación a la fábrica, de forma que se disminuyan las filas de esperas que actualmente se presentan.

Es importante resaltar que al dividir un número por 0 se presenta un error. Ello ocurre a la hora de iniciar la solución del modelo matemático, en el que el software inicializa las variables en 0 , lo que 
genera que la confiabilidad sea 0 y se genere una división por 0 en la función objetivo. Por tal motivo, se le sumó 1 a la confiabilidad. Esto genera que en vez de iniciar la solución de la función costos/ confiabilidad en $0 / 0$, lo haga con $0 / 1$ y se elimina el error de dividir por 0 .

En el escenario de maximizar la confiabilidad, se determinó una confiabilidad de $41,4 \%$ con un costo exageradamente alto, lo que se debe a que no se tienen en cuenta los costos para tomar las decisiones. Solo se tiene en cuenta la maximizar la confiabilidad.

Este valor representa en las condiciones propuestas, la confiabilidad máxima que puede alcanzar la cadena de suministro, pero esta requerirá asumir una diferencia de costos mucho más elevada que en los casos 1 y 2. Por tal motivo, solo se tiene para comparar la confiabilidad máxima con la actual y las encontradas en los otros casos.

Es importante resaltar que los resultados obtenidos del modelo propuesto bajo la relación costos/ confiabilidad tienen cuatro principales beneficios:

- Disminución de las cantidades de envíos, 4 por semana, que al año son 208.

- Aumenta la confiabilidad de la cadena. La probabilidad que no ocurra una falla en el sistema se aumenta, ello debido a que se tiene un incremento de $9,5 \%$ con respecto al estado actual donde la confiabilidad es de $17,7 \%$. Para el caso de minimizar costos se tiene un incremento de 7,5\%.

- Permite a la fábrica controlar el orden de los envíos de cada camión saliente de los centros de consolidación.

- Mejora la robustez de la cadena de suministro, debido a que aumentará a probabilidad de no falla del sistema. Es decir, la cadena de suministro tendrá mayor capacidad de absorber el impacto negativo de ciertos eventos disruptivos.

Finalmente, se puede decir que el modelo selecciona las localizaciones óptimas de los centros de consolidación con base en el objetivo que la empresa considere, ya sea minimizar la división entre costos/confiabilidad, minimizar costo o maximizar la confiabilidad. Para el caso 1 como el 2, se da apertura a los centros de consolidación de las fincas 16 (Santo Tomás) y 18 (Caracolí).
Los autores recomiendan el uso del modelo utilizando el enfoque del caso 1, donde se busque minimizar la relación costos/confiabilidad, ya que esto permitirá que la decisión de seleccionar las instalaciones, además de tener un bajo costo, posea una alta confiabilidad. Se aclara que al utilizar estos dos criterios, la solución no necesariamente es la que genera menos costos ni la que presente la mayor confiabilidad.

En futuras investigaciones, debido que en esta se definió la confiabilidad de la cadena de suministro como la probabilidad de que todos los nodos y arcos de la cadena funcionen al mismo tiempo, se hace interesante la elaboración de modelos matemáticos que permitan determinar la localización óptima de las instalaciones, teniendo en cuenta la importancia que la empresa estudiada quiera darle a los costos y a la confiabilidad en una relación porcentual donde la suma de estas importancia den $100 \%$.

\section{AGRADECIMIENTOS}

Los autores desean expresar su agradecimiento al Programa de Innovación en Logística y Gestión Portuaria del Caribe, Logport, a quien pertenece esta investigación y quien gestionó los recursos financieros para la ejecución de este proyecto por medio del Sistema General de Regalías (SGR) de Colombia, en la línea de ciencia, tecnología e innovación.

\section{REFERENCIAS}

[1] W. Ardila, D. Romero y F. González. "Estrategias para la Gestión de Riesgos en la Cadena de Suministros". Latin American and Caribbean Conference for Engineering and Technology. Guayaquil, Ecuador. 22-24 de julio 2014.

[2] S. Ponomarov and M. Holcomb. "Understanding the concept of supply chain resilience". The International Journal of Logistics Management. Vol. $20 \mathrm{~N}^{\circ}$ 1, pp. 124-143. 2009. DOI: $10.1108 / 09574090910954873$

[3] C. Zobel. "Comparative visualization of predicted disaster resilience". 7th International Conference on Information Systems for Crisis Response and Management - ISCRAM 2010. Washington, Estados Unidos. 2 de mayo de 2010. 
[4] R. Francis and B. Bekera. "A metric and frameworks for resilience analysis of engineered and infrastructure systems". Reliability Engineering \& System Safety. Vol. 121, pp. 90-103. Enero 2014. DOI: 10.1016/j.ress.2013.07.004

[5] W. Klibi, A. Martel and A. Guitouni. "The design of robust value-creating supply chain networks: a critical review". European Journal of Operational Research. Vol. $203 \mathrm{~N}^{\circ} 2$, pp. 283-293. Junio 2010. DOI: 10.1016/j. ejor.2009.06.011

[6] R. Zanjirani, S. Rezapour, T. Drezner and S. Fallah. "Competitive supply chain network design: An overview of classifications, models, solution techniques and applications". Omega. Vol. 42, pp. 92-118. Junio 2014. DOI: $10.1016 /$ j.omega.2013.08 .006

[7] D. Simchi-Levi, P. Kaminsky and E. SimchiLevi. "Managing the supply chain: the definitive guide for the business professional". McGraw Hill. Boston, Estados Unidos. 2004. ISBN: 978-0-07-143587-1.

[8] A. Klose and A. Drexl. "Facility location models for distribution system design". European Journal of Operational Research. Vol. $162 \mathrm{~N}^{\circ} 1$, pp. 4-29. Abril 2005. DOI: 10.1016/j.ejor.2003.10.031.

[9] J. Birge and F. Louveaux. "Introduction to Stochastic Programming". Springer, 2011. DOI: 10.1007/978-1-4614-0237-4. ISBN: 978-1-4614-0236-7.

[10] P. Kouvelis and G. Yu. "Robust Discrete Optimization and its Applications". Springer. Vol. 14. 1997. DOI: 10.1007/978-1-47572620-6. ISBN: 978-1-4419-4764-2.

[11] S. Chopra and M. Sodhi. "Managing risk to avoid supply-chain breakdown". MIT Sloan Management Review. Vol. $46 \mathrm{~N}^{\circ} 1$, pp. 53-61. Octubre 2004.

[12] G. Gutiérrez, P. Kouvelis and A. Kurawala. "A robustness approach to uncapacitated network design problems". European Journal of Operations Research. Vol. 94 $\mathrm{N}^{\circ} 2$, pp. 362-376. Octubre 1996. DOI: 10.1016/0377-2217(95)00160-3.
[13] K. Stecke \& S. Kumar. "Sources of Supply Chain Disruptions, Factors that Breed Vulnerability, and Mitigating Strategies". Journal of Marketing Channels. Vol. 16 $\mathrm{N}^{\circ} 3$, pp. 193-226. Junio 2009. DOI: 10.1080/10466690902932551

[14] V. Vashi, C. Bienstock and J. Mentzer. "The use of response surface methodology to optimize logistics". Journal of business logistics. Vol. $16 \mathrm{~N}^{\circ}$ 2, pp. 197-225. 1995. ISSN: 0197-6729.

[15] S. MirHassani, C. Lucas, G. Mitra, E. Messina and C. Poojari. "Computational solution of capacity planning models under uncertainty". Parallel Computing. Vol. 26 No 5, pp. 511-538. Marzo 2000. DOI: 10.1016/ S0167-8191(99)00118-0.

[16] F. Pan and R. Nagi. "Robust supply chain design under uncertain demand in agile manufacturing". Computers \& Operations Research. Vol. $37 \mathrm{~N}^{\circ}$ 4, pp. 668-683. Abril 2010. DOI: 10.1016/j.cor.2009.06.017.

[17] P. Tsiakis, N. Shah and C. Pantelides. "Design of multiechelon supply chain networks under demand uncertainty". Industrial \& Engineering Chemistry Research. Vol. $40 \mathrm{~N}^{\circ} 16$, pp. 35853604. Julio 2001. DOI: 10.1021/ie0100030.

[18] S. Nickel, F. Saldanha-da-Gama and H. Ziegler. "A multi-stage stochastic supply network design problem with financial decisions and risk management". Omega. Vol. $40 \mathrm{~N}^{\circ}$ 5, pp. 511-524. Octubre 2012. DOI: 10.1016/j.omega.2011.09.006.

[19] T. Santoso, S. Ahmed, M. Goetschalckx and A. Shapiro. "A stochastic programming approach for supply chainnetwork design under uncertainty". European Journal of Operations research. Vol. $167 \mathrm{~N}^{\circ} 1$, pp. 96115. Noviembre 2005. DOI: $10.1016 / \mathrm{j}$. ejor.2004.01.046.

[20] A. Santander, J. Amaya, C. Viloria, A. Sierra, I. Saavedra, D. Romero and F. González. "Diseño de cadenas de suministros resilientes". Ediciones de la U. Primera edición, pp. 45-70. Barranquilla, Colombia. ISBN: 978-958-741-441-7. 2014. 